\title{
Neural correlates of tactile hardness intensity perception during active grasping
}

\author{
Ji-Hyun Kim ${ }^{1}$, Junsuk Kim ${ }^{2}$, Jiwon Yeon ${ }^{3}$, Jang-Yeon Park ${ }^{4}$, Dongil Chung ${ }^{\text {Corresp., } 1}$, Sung-Phil Kim ${ }^{\text {Corresp. } 1}$ \\ ${ }^{1}$ Department of Biomedical Engineering, Ulsan National Institute of Science and Technology, Ulsan, Republic of Korea \\ 2 Department of Industrial ICT Engineering, Dong Eui University, Busan, Republic of Korea \\ 3 Department of Psychology, Georgia Institute of Technology, Atlanta, Georgia, United States \\ 4 Department of Biomedical Engineering, Sungkyunkwan University, Suwon, Republic of Korea \\ Corresponding Authors: Dongil Chung, Sung-Phil Kim \\ Email address: dchung@unist.ac.kr, spkim@unist.ac.kr
}

While tactile sensation plays an essential role in interactions with the surroundings, relatively little is known about the neural processes involved in the perception of tactile information. In particular, it remains unclear how different intensities of tactile hardness are represented in the human brain during object manipulation. This study aims to investigate neural responses to various levels of tactile hardness using functional magnetic resonance imaging while people grasp objects to perceive hardness intensity. We used four items with different hardness levels but otherwise identical in shape and texture. Twenty-five healthy volunteers participated in this study. Before scanning, participants performed a behavioral task in which they received a pair of stimuli and they were to report the perceived difference of hardness between them.During scanning, without any visual information, they were randomly given one of the four objectsand asked to grasp it. We found significant blood oxygen-level-dependent (BOLD) responses in the posterior insula in the right hemisphere (rplns) and the right posterior lobe of the cerebellum (rpCerebellum), which parametrically tracked hardness intensity. These responses were supported by BOLD signal changes in the rpCerebellum and rplns correlating with tactile hardness intensity. Multidimensional scaling analysis showed similar representations of hardness intensity among physical, perceptual, and neural information. Our findings demonstrate the engagement of the rpCerebellum and rplns in perceiving tactile hardness intensity during active object manipulation. 


\section{Neural correlates of tactile hardness perception}

\section{2 during active grasping}

3

4

Ji-Hyun Kim ${ }^{1}$, Junsuk Kim², Jiwon Yeon ${ }^{3}$, Jang-Yeon Park ${ }^{4}$, Dongil Chung ${ }^{1}$, Sung-Phil Kim ${ }^{1}$

${ }^{1}$ Department of Biomedical Engineering, Ulsan National Institute of Science and Technology, Ulsan, Republic of Korea

${ }^{2}$ Department of Industrial ICT Engineering, Dong-Eui University, Busan, Republic of Korea

${ }^{3}$ Department of Psychology, Georgia Institute of Technology, Atlanta, Georgia, United States of America

${ }^{4}$ Department of Biomedical Engineering, Sungkyunkwan University, Suwon, Republic of Korea

Corresponding Author:

Sung-Phil Kim ${ }^{1}$

50, UNIST-gil, Ulsan, 44919, Republic of Korea

Email address: spkim@unist.ac.kr

Dongil Chung ${ }^{1}$

50, UNIST-gil, Ulsan, 44919, Republic of Korea

Email address: dchung@unist.ac.kr

\section{Abstract}

While tactile sensation plays an essential role in interactions with the surroundings, relatively little is known about the neural processes involved in the perception of tactile information. In particular, it remains unclear how different intensities of tactile hardness are represented in the human brain during object manipulation. This study aims to investigate neural responses to various levels of tactile hardness using functional magnetic resonance imaging while people grasp objects to perceive hardness intensity. We used four items with different hardness levels but otherwise identical in shape and texture. Twenty-five healthy volunteers participated in this study. Before scanning, participants performed a behavioral task in which they received a pair of stimuli and they were to report the perceived difference of hardness between them. During scanning, without any visual information, they were randomly given one of the four objects and asked to grasp it. We found significant blood oxygen-level-dependent (BOLD) responses in the posterior insula in the right hemisphere (rpIns) and the right posterior lobe of the cerebellum (rpCerebellum), which parametrically tracked hardness intensity. These responses were supported by BOLD signal changes in the rpCerebellum and rpIns correlating with tactile hardness intensity. Multidimensional scaling analysis showed similar representations of hardness intensity among physical, perceptual, and neural information. Our findings demonstrate the 
39

40

41

42

43

44

45

46

47

48

49

50

51

52

53

54

55

56

57

58

59

60

61

62

63

64

65

66

67

68

69

70

71

72

73

74

75

76

77

engagement of the rpCerebellum and rpIns in perceiving tactile hardness intensity during active object manipulation.

\section{Introduction}

Tactile sensation with the hands is essential for humans to manipulate objects in their daily lives. When manipulating objects with various shapes and textures, humans sense the objects' physical properties via tactile information processing, beginning with interactions between sensory receptors underneath the skin and the object (Skedung et al., 2013). Not only does tactile sensation play an important role in the perception of an object's physical properties but it also facilitates precise object manipulation through sensory feedback (Augurelle et al., 2002; Monzée, Lammarre \& Smith, 2003).

Much effort has been devoted to elucidating the fundamental elements of tactile perception. By and large, human tactile perception can be characterized by four fundamental perceptual dimensions: roughness/smoothness, stickiness/slipperiness, warmth/coolness, and hardness/softness (Hollins et al., 2000; Bensmaïa \& Hollins, 2005). As for tactile roughness perception, the spatial patterns of skin deformation play a key role in the discrimination of roughness (S. J. Lederman \& Taylor, 1972). Particularly, simulated temporal cues (Cascio \& Sathian, 2001; Gamzu \& Ahissar, 2001) as well as tangential forces are known to be important in determining roughness intensity (Smith et al., 2002). As for stickiness perception, its magnitude closely correlates with a parameter of kinetic friction between the skin and a surface (Smith \& Scott, 1996). The perceived magnitude of warmth or coolness is determined by contact coefficients and the heat conducted on the skin area (Ho \& Jones, 2006; Ho \& Jones, 2008; Stevens \& Marks, 1979). Lastly, as for hardness perception, both tactile and kinesthetic information are necessary to discriminate hardness levels (Srinivasan \& LaMotte, 1995). A number of functional magnetic resonance imaging (fMRI) studies have explored tactile information processing in the human brain. Several brain regions including the primary somatosensory area (SI), supplementary motor area, and bilateral temporal poles (Kim et al., 2015) are known to be involved in the perception of tactile roughness. In addition, the secondary somatosensory area (SII) was found to be activated with high-frequency stimulus (Seri et al., 2019; Seri et al., 2020), while the parietal operculum lobe is reportedly engaged in the estimation of surface roughness (Kitada et al., 2005; Stilla \& Sathian, 2008), and the posterior insula shows haptic selectivity in processing visuo-haptic stimuli (Stilla \& Sathain, 2008). With respect to stickiness, neural responses to sticky stimuli were found in the contralateral SI and ipsilateral dorsolateral prefrontal cortex (Yeon et al., 2017), and particularly those in the posterior parietal regions were found to discriminate stickiness intensity (Kim et al., 2017). Furthermore, neural activity in the dorsal posterior insula is correlated with thermal sensation of low temperature (Craig et al., 2000, Peltz et al., 2011; Oi et al., 2017). Recently, it was confirmed that the tactile network, including SI and SII, was activated during detection of tactile gratings using an advanced tactile stimulus that makes a grating using electro-static friction (Voung et al., 2020). 
78 Among all tactile perceptual dimensions, this study aims to investigate neural activity related to

79 hardness. Because tactile hardness is defined by the ratio between the relative force exerted on

80 the surface and its displacement, one can posit that tactile hardness perception relies on

81 proprioception (Okamoto, Nagano \& Yamada, 2013). However, several studies have shown that

82 tactile cues are more attributed to the perception of cutaneous hardness than proprioception

83 (Srinivasan \& LaMotte, 1995; Bergmann Tiest, 2010). As for the neural substrates of tactile

84 hardness perception, a non-human primate study showed that surface compliance modulated the

85 activity of slowly adapting type-1 afferents, but not rapidly adapting afferents (Srinivasan \&

86 LaMotte, 1995). Other studies in non-human primates revealed that neural activities in areas $3 \mathrm{~b}$

87 and 1 in SI could discriminate hard and soft objects (Randolph \& Semmes, 1974) and that

88 lesions in the SII decreased discrimination performance between hard and soft stimuli (Murray \&

89 Mishkin, 1984). In humans, a few studies have shown the involvement of the SI and the parietal

90 operculum, such that cerebral blood volume in these regions increased during hardness sensation.

91 Moreover, the neural activity from the parietal operculum predicts different levels of object

92 softness (Bodegård et al., 2003) during passive touch (Kitada et al., 2019). To date, converging

93 evidence suggests the involvement of the bilateral parietal operculum in perceiving tactile

94 hardness along with other sensory information (Lederman et al., 2001; Reed, Shoham \&

95 Halgren, 2004; Kim et al., 2019).

96 However, it is still unknown how tactile hardness intensity information is processed in the

97 human brain during active exploration. Hence, this study aimed to investigate neural

98 representations of tactile hardness intensity while grasping an object with the hand. In order to

99 recognize an object's hardness, both cutaneous tactile cues and dexterous manipulation applying

100 pressure to an object are needed (Bergmann Tiest, 2010). This active exploration process is

101 difficult to control but nevertheless an important factor in hardness perception.

102 Previous studies have shown that pressing or grasping an object is needed to perceive its

103 hardness (Harper \& Stevens, 1964; Turvey \& Carello, 1995) and that active exploration could

104 improve identification of the tactile properties of an object over passive exploration (Gibson,

105 1962). As such, we expected a more natural identification of the neural correlates of the

106 perception of tactile hardness during active tactile exploration.

107 The hypothesis of this study is that SI and SII neural responses would represent hardness

108 intensities during active grasp exploration. To this end, we employed a dexterous grasping task

109 to elicit hardness sensation (Lederman et al., 2001) and attempted to identify the brain regions

110 that track tactile hardness intensity. Participants performed both psychophysical and fMRI

111 experiments: the psychophysical experiment was designed to measure perceived differences in

112 tactile hardness intensities between stimuli, and the fMRI experiment was designed to measure

113 the neural responses to different hardness intensities.

114

115 Materials \& Methods

116 Participants and ethics approval 
117 Twenty-five healthy volunteers (mean age $=24 \pm 4$ years old, age range $=19-29,9$ male and 16

118 female), with no contraindications against MRI and no history of neurological disorders,

119 participated in this study. All participants were right-handed and had no deficits in tactile

120 processing. Experimental procedures were approved by the ethics committee of the Ulsan

121 National Institute of Science and Technology (UNISTIRB-15-16-A). The study was conducted

122 in accordance with the Declaration of Helsinki. All participants were informed of the study

123 objectives and experimental procedures and voluntarily submitted a written consent form.

\section{Tactile stimuli}

125 The tactile stimuli for the current study had the same physical properties (e.g., shape and surface

126 texture) except their hardness intensity. To satisfy this criterion, we selected a commercially

127 available product (Eggsercizer, Magister Corp.; Chattanooga, TN), primarily used in motor

128 rehabilitation (e.g., grip strengthening). The Eggsercizer is made of synthetic rubber and silicon

129 and has an oval shape with a diameter of $5.1 \mathrm{~cm}$ and a height of $7 \mathrm{~cm}$ (Fig. 1A and 1B). The

130 stimulus weights were approximately identical $(75.24 \pm 0.4 \mathrm{~g})$. The physical hardness intensities

131 were quantitatively measured by resistance to indentation using a Durometer Hardness OO-Type

132 device (ASKER/Shore; OO-Type) at KOPTRI (Korea Polymer Testing \& Research Institute,

133 Seoul, South Korea). For the experiment, four different stimuli with different hardness intensities

134 (28, 36, 45, and 57 a.u.) were prepared where a higher number indicated a harder stimulus

135 (physical hardness intensity of each stimulus is denoted as $\mathrm{H} 28, \mathrm{H} 36, \mathrm{H} 45$, and $\mathrm{H} 57$, hereafter).

136 Although these intensity values did not map exactly to perceived hardness, in general, tactile

137 hardness intensities of 20 and 50 were reportedly perceived similar to those of chewing gum and

138 solid truck tires, respectively. The stimuli were kept clean after each experiment using a wet

139 tissue until their surface was free of matter.

140 Experimental procedure

141 Before the fMRI experiment, participants performed a behavioral task in which they received a

142 pair of stimuli with eyes closed (Fig. 1C). During the behavioral experiment, participants sat on a

143 chair and put their right hands on a desk in front of the chair. To eliminate any visual effects,

144 participants were blindfolded during the entire behavioral experiment. An experimenter placed

145 the stimulus on the palm of the participants' right hand with the thin side oriented toward the

146 thumb. The stimulus was placed at the palm center with the major axis perpendicular to the

147 fingers except the thumb and participants grasped each stimulus once for $1 \mathrm{~s}$ and released it.

148 Participants were instructed to exert a grasping force as constantly as possible for every stimulus.

149 Immediately after participants released the stimulus, the experimenter removed it from

150 participants' hands and placed the next stimulus after a 5-s inter-stimulus interval. In the

151 instruction session, an experimenter presented to participants a pair of stimuli with the highest

152 (H57) and lowest intensities (H28) to allow participants to perceive the greatest difference in the

153 hardness intensity of the experimental stimuli. Then, a pair of stimuli with the same hardness

154 intensity (randomly selected out of four levels) was presented to participants along with

155 information about the same hardness intensity. 
156 Participants performed four main sessions of the behavioral task in each of which a total of 10

157

158

159

160

161

162

163

164

165

166

167

168

169

170

171

172

173

174

175

176

177

178

179

180

181

182

183

184

185

186

187

188

189

190

191

192

193

194

195

stimulus pairs were presented to participants. This set of 10 pairs consisted of pairs of stimuli with different hardness and four additional pairs with the same hardness level $(4 \mathrm{C} 2+4=10$ pairs). In each trial of the behavioral task, participants were presented with one pair of stimuli after the other and grasped each stimulus as described above. Then, they verbally reported a perceived difference. Each participant was allowed to report a difference in their own numerical scale, which would be normalized in the following data analyses (see Data analysis for normalization details). Each main session began with the instruction session. There was a maximum 60-s break between successive sessions. Overall, a total of 40 pairs (10 pairs $\times 4$ repetitions) were presented to participants during the behavioral experiment.

The objective of the fMRI experiment was to identify brain regions related to tactile hardness perception and neural activities representing the hardness intensity during active exploration of presented objects. As in the behavioral experiment, stimuli with four hardness levels were presented to participants. In addition, participants were given a "sham" stimulus as a control task where they executed the same grasping and releasing motions without holding any objects. During this control task, participants were asked to curl their fingers as if they grasped a virtual object in their hands, following the visual cue. Note that participants were instructed to avoid contacting their fingers with the palm to minimize the potential effects of unnecessary tactile sensations.

During the scanning sessions, participants laid comfortably on the MRI bed, leaving their right palm facing up. Two or three foam cushions were placed in the space between the head and the head coil to fix the participant's head, minimizing movement effects. Participants were instructed to grasp the stimuli with constant force, and to focus on stimuli's hardness during the grasping action. The procedure of each trial is illustrated in Fig. 2. An experimenter stood at the entrance of the magnet bore and presented stimuli along the screen's instruction. After a 15-s baseline period, participants performed the grasping task by following the instructions shown on a visual display. Each trial comprised five 3-s grasping periods, taking $15 \mathrm{~s}$ total (Fig. 2). In each grasping period, the cue "Grab" was presented for $2 \mathrm{~s}$. At the same time, the experimenter placed a stimulus on participants' hands. Each time participants viewed the 'Grab' instruction, they were asked to grasp the stimulus for $1 \mathrm{~s}$ and released it afterwards. After $15 \mathrm{~s}$, the stimulus was collected by the experimenter and participants held a resting position for $9 \mathrm{~s}$ until the next trial. The same stimulus was grasped repeatedly by participants in a single trial. A total of five stimuli (four hardness levels plus one sham stimulus) were presented five times each in a fully randomized order over 25 trials per session. Participants conducted two sessions overall. Each session took $618 \mathrm{~s}$ on average.

\section{MRI data acquisition and preprocessing}

The blood oxygen-level-dependent (BOLD) signal of participants during the tactile hardness perception task was measured using a 3-T fMRI scanner (Magnetom TrioTim, Siemens Healthineers; Erlangen, Germany). Three-dimensional (3D) functional images constructed from 48 slices covered the whole cerebrum ( $\mathrm{T} 2 *$-weighted gradient echo planar imaging, covering the

Peerj reviewing PDF | (2021:03:58613:1:1:NEW 4 Jun 2021) 
196

197

198

199

200

201

202

203

204

205

206

207

208

209

210

211

212

213

214

215

216

217

218

219

220

221

222

223

224

225

226

227

228

229

230

231

232

233

234

235

whole depth of brain area, repetition time $[\mathrm{TR}]=3,000 \mathrm{~ms}$, echo time $[\mathrm{TE}]=30 \mathrm{~ms}$, flip angle $=$ $90^{\circ}$, field of view $[\mathrm{FOV}]=192 \mathrm{~mm}$, slice thickness $=3 \mathrm{~mm}$, and voxel size $=2.0 \times 2.0 \times 3.0$ $\mathrm{mm}^{3}$ ). Anatomical high-resolution images were also acquired (T1-weighted 3D magnetization prepared rapid gradient echo sequence, $\mathrm{TR}=2,300 \mathrm{~ms}, \mathrm{TE}=2.28 \mathrm{~ms}$, flip angle $=8^{\circ}, \mathrm{FOV}=$ $256 \mathrm{~mm}$, voxel size $=1.0 \times 1.0 \times 1.0 \mathrm{~mm}^{3}$ ). Functional images were preprocessed using SPM12 software (Wellcome Department of Imaging Neuroscience; London, United Kingdom), following the sequence: slice-timing correction, realignment, co-registration, segmentation, spatial normalization to the Montreal Neurological Institute (MNI) template and smoothing with a 6-mm full-width-half-maximum isotropic Gaussian kernel.

\section{Data analysis}

We excluded four participants from all data analyses because of excessive head movements over the maximal translation ( $2 \mathrm{~mm}, 2 \mathrm{~mm}$, and $3 \mathrm{~mm}$ in $\mathrm{x}, \mathrm{y}, \mathrm{z}$, respectively). Consequently, behavioral and functional data analyses were performed on 21 participants. To estimate the perceptual magnitude of hardness intensity, participants' responses were first normalized into a scale from 0 to 10 using the unity-based normalization given by

$x^{\prime}=\frac{x-\min (x)}{\max (x)-\min (x)} \times 10$

where $\mathrm{x}$ is the original value of participants' responses and $\mathrm{x}^{\prime}$ is the normalized value between 0 to 10. This normalization process was performed on each participant's data.

Then, we examined a possible order effect of stimulus presentation within stimulus pairs (see Behavioral experiment). Specifically, we used a paired t-test to compare the normalized responses between the trials where the same set of stimuli were presented in the opposite order (e.g., [H28, H57] vs. [H57, H28]). In addition, we used a repeated-measures analysis of variance (rmANOVA) to compare the normalized responses among trials involving different levels of tactile hardness.

We performed event-related fMRI analyses of participants' BOLD responses to tactile hardness perception. Two separate design matrices were used for first-level general linear model (GLM) analyses: one design matrix to find brain regions involved in tactile hardness perception, and the other to find the brain regions that track hardness intensity. In the first design matrix, all stimulus types (four hardness levels plus one sham) were modeled as separate linear regressors. We specified tactile perception events as the time at which participants grasped the stimuli with their hand. All events in each regressor were convolved with the canonical hemodynamic response function. At first-level, contrast images that reflected BOLD response differences between all actual versus sham tactile perceptions were generated for each participant. The second-level model was constructed as a one-sample t-test using the contrast images from the first-level. Where indicated, family-wise error (FWE) was controlled to correct for multiple comparisons. In the second design matrix, the subjective hardness intensity obtained from the behavioral experiment was added as a parametric modulator to identify the brain regions that track tactile hardness intensity. At first-level, contrast images that reflected whole-brain responses positively and negatively correlated with hardness intensity were generated for each participant. The same strategy as with the first design matrix (contrast analysis described above) was followed for 
236

237

238

239

240

241

242

243

244

245

246

247

248

249

250

251

252

253

254

255

256

257

258

259

260

261

262

263

264

265

266

267

268

269

270

271

272

273

274

275

second-level analysis and multiple comparisons correction. For both contrast and parametric modulation analyses, we labeled statistically significant clusters using the automated anatomical labeling toolbox (Tzourio-Mazoyer et al., 2002).

Furthermore, we calculated and depicted the percent signal change (PSC) within the brain regions identified by parametric modulation analysis to better illustrate the region's responses tracking the stimuli hardness information. PSC was calculated as the BOLD signal ratio in response to stimuli over that without stimulus. This analysis aimed to supplement the finding of brain regions above by confirming the correlation between the PSC in the identified regions and hardness intensity. We set the activated regions determined by the parametric modulation analysis as regions of interest (ROIs) and estimated the PSC of each single ROI in response to each hardness stimulus using the Marsbar toolbox (Brett et al., 2016).

To examine relationships between physical intensities, perceptual responses, and neural responses regarding tactile hardness, we further constructed the dissimilarity matrices of each data type. The PSC in the ROIs above was used as representational neural responses to tactile hardness. As the perceptual responses were normalized in a scale between 0 and 10, neural and physical intensity data were similarly normalized between 0 and 10 . A dissimilarity matrix of physical hardness was constructed using the normalized differences between every pair of the four tactile stimuli, resulting in a $4 \times 4$ symmetric matrix with diagonal entries equal to zero. We constructed the dissimilarity matrix of perceptual responses in the following manner. First, participants' perceptual responses to each stimulus were normalized within a scale from 0 to 10 using the unity-based normalization given in equation (1). Second, the difference of the normalized perceptual responses between every possible pair of stimuli was calculated. Third, the difference values for each pair were averaged over all participants. Fourth, a dissimilarity matrix of perceptual responses was constructed from each averaged difference. The dissimilarity matrix of neural responses of each ROI was also constructed using the normalized PSC difference values averaged across participants but resulting in a $4 \times 4$ symmetric matrix.

A Mantel test, often used for analyzing functional connectivity in the brain (Glerean et al., 2016), was employed to find statistically significant correlations (Pearson's correlation) between dissimilarity matrices (Mantel, 1967).

Lastly, we applied multidimensional scaling (MDS) to the dissimilarity matrices to find lowdimensional representations of physical, perceptual, and neural responses. These lowdimensional representations of hardness intensity were meant to visualize similarities between the three different domains of tactile hardness: physical, perceptual, and neural representation of object hardness.

\section{Results}

\section{Behavioral responses to tactile stimuli with different hardness intensities}

We first confirmed the lack of order effects in perceived difference between the stimuli set. Thus, we did not further consider presentation order in subsequent analyses of behavioral responses. It also led us to define the absolute perceptual differences between a pair of stimuli as 
276 the perceived distance of tactile hardness. Then, we examined differences in perceived distance

277 among all possible pairs of tactile stimuli using rmANOVA and observed a significant difference 278 of mean perceived distance among those pairs $(\mathrm{F}((9,180))=144.62, \mathrm{p}<0.0001)$ (Fig. 3). A post-

279 hoc Bonferroni test further revealed significant differences in perceived distance between pairs 280 with dissimilar hardness differences (all $p<0.0001$; Fig. 3) but no difference between pairs with 281 similar hardness differences. As an additional explorative analysis, we examined potential 282 gender differences in the perceived distance among all possible pairs of tactile stimuli using two 283 sample t-tests, but none of the pairs showed significant difference between male and female 284 participants (Table S1). These results show that participants could accurately perceive objective 285 differences in hardness between pairs of tactile stimuli.

286 Neural responses to tactile stimuli with different hardness intensities

287 We examined the BOLD signal evoked by tactile hardness by contrasting each hardness intensity

288

289

290

291

292

293

294

295

296

297

298

299

300

301

302

303

304

305

306

307

308

309

310

311

312

313

314

315

against the "Sham" using GLM analysis. However, we found no commonly activated regions across the whole brain. To examine the brain regions that track the physical properties (hardness intensity) of stimuli, we ran a whole-brain analysis using a design matrix including physical hardness intensity as a parametric modulator. We found a significant positive correlation between hardness intensity and neural responses in the posterior insula in the right hemisphere (rpIns) (MNI space coordinates of $\mathrm{x}=40, \mathrm{y}=-26, \mathrm{z}=18$; FWE corrected $\mathrm{p}<0.05$, cluster size $>10$, cluster defining threshold $\mathrm{p}<0.001$ ) (Fig. 4A). A set of brain regions negatively tracked physical hardness intensity (Fig. 5A). And we found a significant negative correlation in the right posterior lobe of the cerebellum (rpCerebellum) (MNI space coordinates of $x=42, y=-74, z=$ -40 ; FWE corrected $\mathrm{p}<0.05$, cluster size $>10$, cluster defining threshold $\mathrm{p}<0.001$ ) (Fig. 5A).

\section{Dissimilarity analysis}

We constructed three dissimilarity matrices of physical hardness intensities, perceived responses, and neural responses to hardness intensities (Fig. 6). For the neural dissimilarity matrix, PSC differences between the rpIns and rpCerebellum were used as a neural representation of tactile hardness.

MDS analysis of the dissimilarity matrices produced two-dimensional (2D) representations of physical, neural, and perceptual hardness intensity (Fig. 7). This 2D depiction clearly visualized the response clustering into four groups according to hardness intensity across all three types of representations (physical, perceptual, and neural). Moreover, it appeared that only one dimension (MDS1) was sufficient to effectively represent hardness intensity, not only for physical stimuli properties but also for neural and perceptual responses.

\section{Discussion}

The aim of the current study was to investigate neural responses to various levels of tactile hardness during active object grasping. The behavioral results clearly demonstrated that each stimulus could be distinguished by its physical hardness intensity. We found that the neural responses in the rpIns positively track the perceived tactile hardness intensity, while those of the rpCerebellum negatively track hardness intensity. The low-dimensional representations of 
316 physical hardness, perceptual responses, and neural responses obtained by MDS analysis,

317 commonly revealed a clear segregation of hardness intensity, again supporting that the rpIns and

318 rpCerebellum encode tactile hardness intensity information. To the best of our knowledge, this is

319 the first study to reveal the neural correlates of hardness intensity perception during active tactile

320 exploration in humans.

321 Perceptual discrimination of tactile hardness

322 The behavioral results showed that participants could distinguish different levels of tactile

323 hardness when they grasped the presented stimuli. Self-reported perceptual differences between

324 stimuli well-reflected the relative physical difference of objects' hardness. In our data, stimulus

325 presentation order did not affect the recognition of hardness differences. This might be due to the

326 fact that we only used four different types of stimuli (i.e., the four stimuli resulted in six distinct

327 pairs); participants might have easily learned the minimum and maximum hardness at the

328 beginning of each session which might helped with accurate estimation. Nonetheless, we

329 prepared stimuli with clearly separated hardness for this study because we wanted to explore

330 neural substrates of well explained tactile perception.

331 Brain regions representing tactile hardness intensity

332 By comparing brain responses to stimuli against sham, we attempted to find brain regions

333 involved in hardness intensity perception. Because all stimuli had the same shape and surface

334 texture, we expected to identify the brain regions commonly involved in different hardness

335 intensity perception by contrasting each hardness intensity against the sham trials. However,

336 there was no such commonly responsive brain region, including the SI or SII. A possibility is

337 that the SI might have been involved in sham trials due to participants' imaginary tactile

338 perception. Previous rodent studies showed similar prominent activation in both the MI and SI

339 during motor control (Diamond et al., 2008; Matyas et al., 2010). In our task, hand movements

340 were dominant in all trials because participants perceived hardness via active exploration,

341 leading to SI activations in response to both sham and tactile stimulations.

342 We found that neural responses in the rpIns increased as a function of perceived tactile hardness.

343 Conversely, neural activity in the rpCerebellum decreased with increased perceived tactile

344 hardness. The rpIns has been reported to be involved in tactile pressure and proprioceptive

345 perception. Previous studies reported bilateral posterior insula activation during a tactile hardness

346 perception task (Lederman et al., 2001) and the involvement of the posterior insula in tactile and

347 body displacement perception and bodily self-consciousness (Richer et al., 1993; Blefari et al.,

348 2017; Findlater et al., 2018), as well as activation in response to tactile pressure stimulation

349 (Chung et al., 2015). Based on previous lesion studies, the posterior insula is known to be

350 involved in self-awareness of actions (Karnath et al., 2010). Previous research backs up our

351 finding that rpIns might be engaged in the perception of hardness intensity during active

352 grasping. Our results provide additional evidence that the human posterior insula might play a

353 role in the tactile perception of hardness intensity. While we found activation only in the rpIns, a

354 more liberal statistical threshold ( $p<0.001$, uncorrected) revealed involvement of the bilateral

355 posterior insula. Future studies should examine the functional connectivity between the bilateral 
356

357

358

359

360

361

362

363

364

365

366

367

368

369

370

371

372

373

374

375

376

377

378

379

380

381

382

383

384

385

386

387

388

389

390

391

392

393

394

395

posterior insula and other brain regions, such as the SI, to understand why rpIns showed stronger responses than the contralateral hemisphere.

One of the most noticeable findings in the current study is that the cerebellum was activated during active perception of tactile hardness. A number of human neuroimaging studies has shown that the cerebellum is functionally connected to the sensorimotor network combining the sensorimotor cortex, premotor area, and supplementary motor area. In addition, the cerebellum is known to participate in motor control, motor learning, and motor planning (Habas et al., 2009; Krienen \& Buckner, 2009; O’Reilly et al., 2010; Zeeuw \& Brinke., 2015; Gao et al., 2018). Particularly, the rpCerebellum is known to be engaged in self-produced tactile perception (Blakemore, Wolper \& Frith, 1999), which may be necessary in to recognize hardness intensity. According to a recent study, functional connectivity between the primary somatosensory cortex and the cerebellum was strengthened when participants reported perceptual attenuations of selfgenerated touch (Kilteni \& Ehrsson, 2020). These results suggest that the ipsilateral cerebellum has a crucial role in predicting self-generated touch. Our results showed that cerebellar activity is associated with perceived tactile hardness during active grasping, and are in line with the aforementioned previous findings.

\section{Relationship between neural responses, physical, and perceptual tactile hardness}

The Mantel test and MDS analysis were conducted to intuitively illustrate the relationship among physical, perceptual, and neural representations of tactile hardness intensity. The Mantel test results suggested similar perception of different levels of hardness intensity to neural responses, which were marginally correlated with the physical hardness of stimuli, suggesting that the brain tracks the subjective perception of objects' hardness rather than their physical hardness. The MDS analysis showed similar clustering of physical, perceptual, and neural representations of each hardness level. This confirms that neural responses in both posterior insula and cerebellar regions represented perceived hardness.

\section{Limitations}

This study has a few limitations. First, we assumed that individuals show stable perceptual responses. In the behavioral experiments, we could identify the relationship between the physical and the perceived hardness for each participant. During the scanning phase, we did not directly collect participants' subjective responses because we intended for participants to fully concentrate on the grasping motions. Under our experimental environment, it was difficult to obtain a direct response from participants during MRI scanning. During neural image acquisition, participants explored stimuli with their right hand and held a squeeze ball for emergency with their left hand, which prohibited them from making behavioral responses with button pressing. Alternatively, we attempted to receive verbal responses directly during scanning, but the scanner noise was too loud. As such, we decided to separate behavioral and neural imaging experiments while ensuring that participants maintained the same active grasping across both experiments. Therefore, the question whether the neural activations measured in the scanner would be the same as those outside remains unanswered. Similarly, albeit very unlikely, it is still unclear whether participants experienced the same hardness perception outside the 
396

397

398

399

400

401

402

403

404

405

406

407

408

409

410

411

412

413

414

415

416

417

418

419

420

421

422

423

424

425

426

427

428

429

430

431

432

433

434

435

scanner as inside. To minimize this difference, we trained participants to grasp the stimuli with constant force before conducting the main experiments. Future studies could use MRIcompatible electromyography (EMG) or force sensors that can measure individuals' grasping force during the scanning phase.

Second, our findings may be limited by the fact that only four hardness intensities were used. We observed that neural responses in the cerebellum and posterior insula were correlated with perceived hardness. However, due to the small number of stimulus types, our data cannot distinguish subtle differences between models that assume linear versus non-linear (e.g., logarithmic, quadratic) associations of neural and perceptual responses.

Third, there is a chance that the neural responses measured in this study might also reflect the changing reaction force applied to the hand with hardness. To minimize this confounding effect, we intended to ensure that participants grasped the stimuli with a constant force. However, it is still impossible to determine whether participants exerted exactly the same force during the experiment. In an effort to bypass this factor, we used subjective ratings of hardness levels rather than physical hardness, so that the self-reported rates should already take the participants' own perception of exerted force into account. Still, to further optimize the task design, future studies could use a visual feedback that shows an EMG-based gage to inform participants of their exerted force in real-time. In addition, in an experiment with real-time EMG, we could observe neural responses during tactile information processing using EEG or MEG with finer time resolution (Golnaz et al., 2020; Hagiwara et al., 2020), which would help confirm the relationship between muscle strength and neural responses in real-time.

Fourth, our experimental design solely focused on active exploration of tactile stimuli. Thus, potential individual differences in passive tactile perception may exist. Note that we controlled for such a confounding factor by examining neural responses parametrically modulated as a function of perceived stimulus hardness.

Fifth, and lastly, it is possible that the marginal association between neural activity and physical hardness could be partially due to insufficient statistical power (the sample size in this study was pre-determine based on that used in similar studies using functional neuroimaging in tactile perception (Laura, 2016)). Our task design and instruction do not rule out the possibility that neural responses would encode physical hardness, but our results suggest that the neural responses elicited in our active grasping task are more associated with perceptual object discrimination rather than tracking of physical hardness per se.

Also, from our explorative analyses addressing potential gender differences, inconsistent with a previous report (Abdouni et al., 2018), we did not find any differences in either behavioral or neural results. Still, as this is an exploratory approach, the interpretation of this result should be cautious. Such an inconsistency could be due to lack of power, given our small sample size for each gender group, and thus, future studies would be necessary to further examine the potential effects of gender differences in tactile sensation.

\section{Conclusions}


436 Nevertheless, in the present study, we found several brain regions associated with hardness 437 intensity perception during active exploring. Neural responses in the cerebellum and posterior 438 insula regions significantly correlated with physical hardness intensity and behavioral intensity 439 rating. Extension of the tactile dimensions (e.g., slipperiness) and exploration of a broader 440 psychophysical range in future studies may further our understanding of human tactile 441 information processing. Further, by using multivoxel pattern analysis and functional connectivity 442 analyses, we can construct more sophisticated neural and behavioral models in tactile processing 443 that may provide insights for developing realistic haptic devices and artificial intelligence that 444 simulates human's subjective tactile perception.

445

446

\section{Acknowledgements}

447 The authors would like to thank Enago (www.enago.co.kr) for the English language review.

448

449

450

\section{References}

Abdouni, A., Moreau, G., Vargiolu, R. \& Zahouani, H. S. 2018. Static and active tactile

451 perception and touch anisotropy: aging and gender effect. Scientific Reports 8, 14240

452

453

454

455

456

457

458

459

460

461

462

463

464

465

466

467

468

469

470

471

472

473

474

475

Augurelle, A.-S., Penta, M., White, O., \& Thonnard, J.-L. 2002. The effects of a change in gravity on the dynamics of prehension. Journal of Gravitational Physiology 9(1):51-3 https://doi.org/10.1007/s00221-002-1322-3

Bensmaïa, S., \& Hollins, M. 2005. Pacinian representations of fine surface texture. Perception \& Psychophysics 67(5):842-854 https://doi.org/10.3758/BF03193537.

Bergmann Tiest, W. M. 2010. Tactual perception of material properties. Vision Research 50(24):2775-2782 https://doi.org/10.1016/J.VISRES.2010.10.005.

Blakemore, S.-J., Wolpert, D. M., \& Frith, C. D. 1999. The Cerebellum Contributes to Somatosensory Cortical Activity during Self-Produced Tactile Stimulation. NeuroImage 10(4):448-459 https://doi.org/10.1006/NIMG.1999.0478.

Blefari, M. L., Martuzzi, R., Salomon, R., Bello-Ruiz, J., Herbelin, B., Serino, A., \& Blanke, O. 2017. Bilateral Rolandic operculum processing underlying heartbeat awareness reflects changes in bodily self-consciousness. European Journal of Neuroscience 45(10):1300-1312. https://doi.org/10.1111/ejn.13567.

Bodegård, A., Geyer, S., Herath, P., Grefkes, C., Zilles, K., \& Roland, P. E. 2003. Somatosensory areas engaged during discrimination of steady pressure, spring strength, and kinesthesia. Human Brain Mapping 20(2):103-115. https://doi.org/10.1002/hbm.10125. 
476 Brett, M., Anton, J.-L., Valabregue, R., \& Poline, J.-B. 2016. MarsBaR region of interest toolbox 477 for SPM. Available at http://marsbar.sourceforge.net (accessed 10 October 2017).

478

479

Brodoehi, S., Gaser, C., Dahnke, R., Witte, W. W. \& Klingner, C. M. 2020. Surface-based

480

481

482

483

484

485

486

487

488

489

490

491

492 analysis increases the specificity of cortical activation patterns and connectivity results. Scientific Reports. 10: 5737 https://doi.org/10.1038/s41598-020-62832-z

Cascio, C. J., \& Sathian, K. 2001. Temporal cues contribute to tactile perception of roughness. Journal of Neuroscience 21(14):5289-96 https://doi.org/10.1523/JNEUROSCI.21-14$\underline{05289.2001 .}$

Case, L. K., Laubacher, C. M., Olausson, H., Wang, B., Spagnolo, P. A. \& Bushnell, M. C. Encoding of Touch Intensity But Not Pleasantness in Human Primary Somatosensory Cortex. 2016. Journal of Neuroscience 36(21):5850-5860 https://doi.org/10/1523/JNEUROSCI.113015.2016

493

494

495

496

497

498

499

500

501

502

503

504

505

506

507

508

509

510

511 Gao, Z., Davis, C., Thomas, A. M., Economo, M. N., Aberego, A. M., Svoboda, K., Zeeuw, C. I.

512 D. \& Li, N. 2018. A cortico-cerebellar loop for motor planning. Nature 563:113-116

513 https://doi.org/10.1038/s41586-018-0633-x

514

515

516 Gibson, J. J. 1962. Observations on active touch. Psychological Review 69(6):477-491

517 https://doi.org/10.1037/h0046962. 
518

519 Glerean, E., Pan, R. K., Salmi, J., Kujala, R., Lahnakoski, J. M., Roine, U., ... Jääskeläinen, I. P. 520 2016. Reorganization of functionally connected brain subnetworks in high-functioning autism.

521

522

523

524

525

526

527

528

529

530

531

532

533

534

535

536

537

538

539

540

541

542

543

544

545

546

547

548

549

550

551

552

553

554

555

556

557

558 Human Brain Mapping 37(3):1066-1079 https://doi.org/10.1002/hbm.23084.

Golnaz, B., Mahmood, A., Falotico, E., \& Laschi, C. 2020 Recurrence quantification analysis of EEG signals for tactile roughness discrimination. International Journal of Machine Learning and Cybernetics 12(4):1-22 https://doi.org/10.1007/s13042-020-01224-1.

Habas, C., Kamdar, N., Nguyen, D., Prater, K., Beckmann, C. F., Menon, V., \& Greicius, M. D. 2009. Distinct cerebellar contributions to intrinsic connectivity networks. Journal of Neuroscience 29(26):8586-94 https://doi.org/10.1523/JNEUROSCI.1868-09.2009.

Hagiwara, K., Ogata, K., Hironaga, N., \& Tobimatsu, S. 2020. Secodnary somatosensory area is involved in vibrotactile temporal-structure processing: MEG analysis of slow cortical potential shifts in humans. Somatosensory \& Motor Research 37(3):222-232 https://doi.org/10.1080/0899 0220.2020 .1784127$.

Harper, R., \& Stevens, S. S. 1964. Subjective Hardness of Compliant Materials. Quarterly Journal of Experimental Psychology 16(3):204-215

https://doi.org/10.1080/17470216408416370.

Ho, H.-N., \& Jones, L. A. 2006. Contribution of thermal cues to material discrimination and localization. Perception \& Psychophysics 68(1):118-128 https://doi.org/10.3758/BF03193662.

Ho, H.-N., \& Jones, L. A. 2008. Modeling the thermal responses of the skin surface during hand-object interactions. Journal of Biomechanical Engineering 130(2):021005 https://doi,org/ 10.1115/1.2899574.

Hollins, M., Bensmaïa, S., Karlof, K., \& Young, F. 2000. Individual differences in perceptual space for tactile textures: Evidence from multidimensional scaling. Perception \& Psychophysics 62(8):1534-1544 https://doi.org/10.3758/BF03212154.

Hu, L., Zhang, L., Chen, R., Yu, H., Li, H. \& Mouraux, A. 2015. The primary somatosensory cortex and the insula contribute differently to the processing of transient and sustained nociceptive and non-nociceptive somatosensory inputs. Human brain mapping 36(11):4346-4360 https:doi.org/10.1002/hbm.22922

Karnath, H. O. \& Baier, B. 2010. Right insula for our sense of limb ownership and selfawareness of actions. Brain Structure and Function 214:411-417 https://doi.org/10.1007/s00429010-0250-4 
559

560

561

562

563

564

565

566

567

568

569

570

571

572

573

574

575

576

577

578

579

580

581

582

583

584

585

586

587

588

589

590

591

592

593

594

595

596

597
Kilteni, K. \& Ehrsson, H. H. 2020. Functional Connectivity between the Cerebellum and Somatosensory Areas Implements the Attenuation of Self-Generated Touch. Journal of Neuroscience 40(4):894-906 https://doi.org/10.1523/JNEUROSCI.1732-19.2019

Kim, J., Chung, Y. G., Park, J.-Y., Chung, S.-C., Wallraven, C., Bülthoff, H. H., \& Kim, S.-P. 2015. Decoding Accuracy in Supplementary Motor Cortex Correlates with Perceptual Sensitivity to Tactile Roughness. PLOS ONE 10(6):e0129777 https://doi.org/10.1371/journal.pone.0129777.

Kim, J., Yeon J, Ryu J, Park J-Y, Chung S-C, Kim S-P. Neural activity patterns in the human brain reflect tactile stickiness perception. Frontiers in Human Neuroscience. 2017;11:445 https://doi.or/ 10.3389/fnhum.2017.00445

Kim, Y., Usui, N., Miyazaki, A., Haji, T., Matsumoto, K., Taira, M., Nakamura, K., \& Katsuyama, N. 2019. Cortical Regions Encoding Hardness Perception Modulated by Visual Information Idnetified by Functional Magnetic Resonance Imaging with Multivoxel pattern Analysis. Frontiers in Systems Neuroscience 13:52 https://doi.org/10.1007/s13042-020-01224-1

Kitada, R., Doizaki, R., Kwon, J., Tanigawa, T., Nakagawa, E., Kochiyama, T., ... Sadato, N. 2019. Brain networks underlying tactile softness perception: A functional magnetic resonance imaging study. NeuroImage 197:156-166 https://doi.org/10.1016/j.neuroimage.2019.04.044.

Kitada, R., Hashimoto, T., Kochiyama, T., Kito, T., Okada, T., Matsumura, M., ... Sadato, N. 2005. Tactile estimation of the roughness of gratings yields a graded response in the human brain : an fMRI study. NeuroImage 25:90-100

https://doi.org/10.1016/j.neuroimage.2004.11.026.

Krienen, F. M., \& Buckner, R. L. 2009. Segregated Fronto-Cerebellar Circuits Revealed by Intrinsic Functional Connectivity. Cerebral Cortex 19(10):2485-2497

https://doi.org/10.1093/cercor/bhp135.

Lederman, S., Gati, J., Servos, P., \& Wilson, D. 2001. fMRI-derived cortical maps for haptic shape, texture, and hardness. Cognitive Brain Research 12(2):307-313

https://doi.org/10.1016/S0926-6410(01)00041-6.

Lederman, S. J., \& Taylor, M. M. 1972. Fingertip force, surface geometry, and the perception of roughness by active touch. Perception \& Psychophysics 12(5):401-408 https://doi.org/10.3758/BF03205850. 
598 Lelic, D., Nissen, T. D., Brock, C., Aziz, Q. \& Drewes, A. M. 2015. Rapid balloon distension as 599 a tool to study cortical processing of visceral sensations and pain. Neurogastroenterology \& 600 Motility 27(6):832-840 https://doi.org/10.1111/nmo.12557

601

602 Mantel, N. 1967. The Detection of Disease Clustering and a Generalized Regression Approach. 603 Cancer Research, 27(2):209-220.

604

605 Matyas, F., Sreenivasan, V., Marbach, F., Wacongne, C., Barsy, B., Mateo, C., ... Petersen, C. 606 C. H. 2010. Motor control by sensory cortex. Science 330(6008):1240-1243

607 https://doi.org/10.1126/science.1195797.

608

609

610

Monzée, J., Lamarre, Y., \& Smith, A. M. 2003. The Effects of Digital Anesthesia on Force

611 Control Using a Precision Grip. Journal of Neurophysiology 89(2):672-683

612

613 https://doi.org/10.1152/jn.00434.2001.

Murray, E. A., \& Mishkin, M. 1984. Relative contributions of SII and area 5 to tactile

614 discrimination in monkeys. Behavioural Brain Research 11(1):67-83

615 https://doi.org/10.1016/0166-4328(84)90009-3.

616

617 O’Reilly, J. X., Beckmann, C. F., Tomassini, V., Ramnani, N., \& Johansen-Berg, H. 2010.

618 Distinct and Overlapping Functional Zones in the Cerebellum Defined by Resting State

619 Functional Connectivity. Cerebral Cortex 20(4):953-965 https://doi.org/10.1093/cercor/bhp157.

620

621 Oi, H., Hashimoto, T., Nozawa, T., Kanno, A., \& Kawata, N. 2017. Neural correlates of ambient 622 thermal sensation: An fMRI study. Scientific Reports, 7(1):1-11.

623 https://doi.org/10.1038/s41598-017-11802-z

624

625

Okamoto, S., Nagano, H., \& Yamada, Y. 2013. Psychophysical dimensions of tactile perception

626 of textures. IEEE Transactions on Haptics 6(1):81-93 https://doi.org/10.1109/ToH.2012.32.

627

628

Peltz, E., Seifert, F., DeCol, R., Dörfler, A., Schwab, S., \& Maihöfner, C. 2011. Functional

629 connectivity of the human insular cortex during noxious and innocuous thermal stimulation.

630 NeuroImage 54(2):1324-35 https://doi.org/10.1016/j.neuroimage.2010.09.012.

631

632

Randolph, M., \& Semmes, J. 1974. Behavioral Consequences of Selective Subtotal Ablations in

633 the Postcentral Gyrus of Macca mulatta. Brain Research 70:55-70. https://doi.org/10.1016/0006-

634 8993(74)90211-x

635

636 Reed, C. L., Shoham, S., \& Halgren, E. 2004. Neural substrates of tactile object recognition: An

637 fMRI study. Human Brain Mapping 21(4):236-246 https://doi.org/10.1002/hbm.10162. 
638

639

640

641

642

643

644

645

646

647

648

649

650

651

652

653

654

655

656

657

658

659

660

661

662

663

664

665

666

667

668

669

670

671

672

673

674

675

676

677

678

Richer, F., Martinez, M., Robert, M., Bouvier, G., \& Saint-Hilaire, J.-M. 1993. Experimental Brain Research Stimulation of human somatosensory cortex: tactile and body displacement perceptions in medial regions. Exp Brain Res 93:173-176 https://doi.org/10.1007/BF00227792

Schweisfurth, M. A., Frahm, J, Farina, D. \& Schweizer, R. 2018. Comparison of fMRI Digit Representations of the Dominant and Non-dominant Hand in the Human Primary Somatosensory Cortex. Frontiers in Human Neuroscience 12:492 https://doi.org/fnhum.2018.00492

Seri, F. A. S., Hamid, A. I. A., Abdullah, J. M., Idris, Z., \& Omar, H. 2019. Brain responses to frequency changes due to vibratory stimulation of human fingertips: An fMRI study. Journal of Physics Conf. Ser. 1248012029 https://doi.org/10.1088/1742-6596/1248/1/012029.

Seri, F. A. S., Hamid, A. I. A., Abdullah, J. M., Idris, Z., \& Omar, H. 2020. Brain responses to high frequencies $(270-480 \mathrm{~Hz})$ changes due to vibratory stimulation of human fingertips: An fMRI study. Journal of Physics Conf. Ser. 1497012012 https://doi.org/10.1088/1742$6596 / 1497 / 1 / 012012$.

Skedung, L., Arvidsson, M., Chung, J. Y., Stafford, C. M., Berglund, B., \& Rutland, M. W. 2013. Feeling small: Exploring the tactile perception limits. Scientific Reports 3(1):2617 https://doi.org/10.1038/srep02617

Smith, A. M., Chapman, C. E., Deslandes, M., Langlais, J.-S., \& Thibodeau, M.-P. 2002. Role of friction and tangential force variation in the subjective scaling of tactile roughness. Experimental Brain Research 144(2):211-223 https://doi.org/10.1007/s00221-002-1015-y.

Smith, A. M., \& Scott, S. H. 1996. Subjective Scaling of Smooth Surface Friction. Journal of Neurophysiology 75(5):1957-1962 https://doi.org/10.1152/jn.1996.75.5.1957

Srinivasan, M. A., \& LaMotte, R. H. 1995. Tactual discrimination of softness. Journal of Neurophysiology 73(1):88-101 https://doi.org/10.1152/jn.1995.73.1.88

Stevens, J. C., \& Marks, L. E. 1979. Spatial summation of cold. Physiology \& Behavior 22(3):541-547 https://doi.org/10.1016/0031-9384(79)90023-4

Stilla, R., \& Sathian, K. 2008. Selective visuo-haptic processing of shape and texture. Human Brain Mapping 29(10):1123-1138 https://doi.org/10.1002/hbm.20456

Turvey, M. T., \& Carello, C. 1995. Dynamic Touch. In: Perception of Space and Motion Elsevier 401-490 https://doi.org/10.1016/B978-012240530-3/50013-4

Peer) reviewing PDF | (2021:03:58613:1:1:NEW 4 Jun 2021) 
679 Tzourio-Mazoyer, N., Landeau, B., Papathanassiou, D., Crivello, F., Etard, O., Delcroix, N., ... 680 Joliot, M. 2002. Automated Anatomical Labeling of Activations in SPM Using a Macroscopic 681 Anatomical Parcellation of the MNI MRI Single-Subject Brain. NeuroImage 15(1):273-289

682 https://doi.org/10.1006/NIMG.2001.0978

683

684 Vuong, Q. C., Shaaban, A. M, Black C, Smith J, Nassar M, Abozied A, Degenaar, P \& Al685 Atabany, W. 2020. Detection of Simulated Tactile Gratings by Electro-static Friction Show a 686 Dependency on Bar Width for Blind and Sighted Observers, and Preliminary Neural Correlates 687 in Sighted Observers. Frontiers in Neuroscience 14:548030 https://doi.org/10.3389/fnins.2020.

688548030.

689

690 Yeon, J., Kim, J., Ryu, J., Park, J.-Y., Chung, S.-C., \& Kim, S.-P. 2017. Human Brain Activity

691 Related to the Tactile Perception of Stickiness. Frontiers in Human Neuroscience 11:8

692 https://doi.org/10.3389/fnhum.2017.00008

693

694 Zeeuw, C. I. D. \& Brinke, M. M. T. 2015. Motor Learning and the Cerebellum. Cold Spring

695 Harb Perspect Biology 7(9):a021683 https://doi.org/10.1101/cshperspect.a021683

696 


\section{Figure 1}

Stimuli and behavioral experiment procedure.

(A) Grasping posture during experiment. (B) Each stimulus featured one of the four hardness intensities measured by the durometer: $28,36,45$, and 57 . The stimuli used in this study are commercially available (Eggsercizer, Magister Corp.; Chattanooga, USA). The stimuli were made of synthetic rubbers. Each stimulus had an identical oval shape with a 5.1-cm diameter and 7-cm height. (C)The behavioral task was conducted before the fMRI experiment. Participants were asked to report perceived hardness differences between each provided pair of stimuli. A set of ten pairs of stimuli was provided to participants, which was repeated four times.
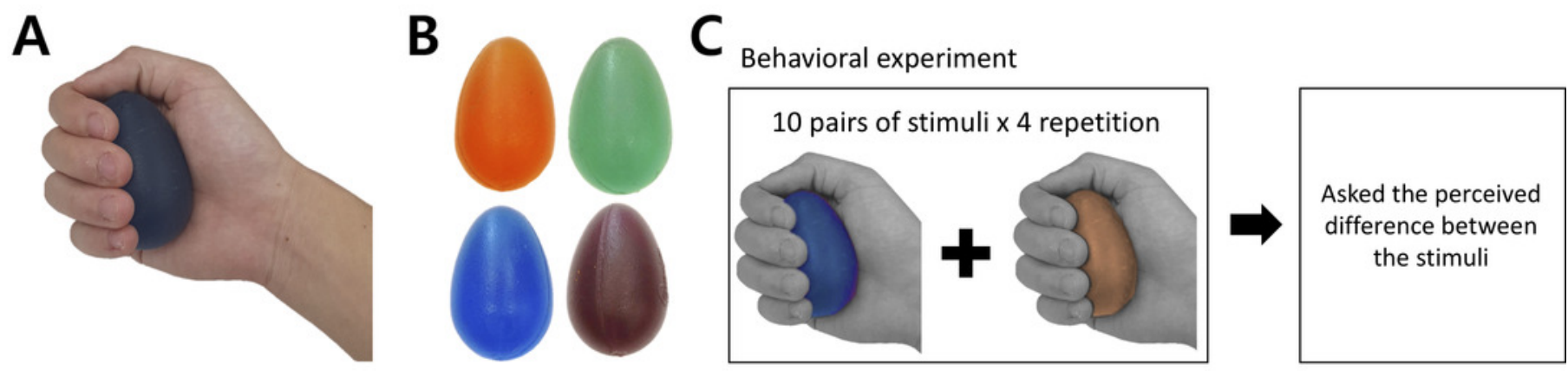
Figure 2

fMRI experiment process

The fMRI task consisted of 25 active grasping and resting trials in an interleaved order; each grasping trial comprised five 3-s grasping periods where participants were asked to grab each stimulus for one second and release it for the subsequent two seconds. In each grasping period, participants were instructed to follow the visual cues of instructions presented on the screen.

\begin{tabular}{|c|c|c|c|c|c|c|c|}
\hline $2 \mathrm{~s}$ & $2 \mathrm{~s}$ & $2 \mathrm{~s}$ & $2 \mathrm{~s}$ & $2 \mathrm{~s}$ & & & \\
\hline Grab & Grab & Grab & Grab & Grab & & Rest & \\
\hline $1 \mathrm{~s}$ & $1 \mathrm{~s}$ & $1 \mathrm{~s}$ & $1 \mathrm{~s}$ & $1 \mathrm{~s}$ & & 9s & \\
\hline $3 s$ & $3 s$ & $3 \mathrm{~s}$ & $3 \mathrm{~s}$ & $3 s$ & & Rest & \\
\hline & $15 \mathrm{~s}$ & & 9s & & $15 \mathrm{~s}$ & & 9s \\
\hline & Trial 1 & & Rest & & Trial 25 & & Rest \\
\hline
\end{tabular}


Figure 3

Behavioral experiment results

Participants were able to perceive the objective hardness level differences between the paired stimuli. Error bars indicate s.e.m. ${ }^{* * *}$ p $<0.001 ;$ n.s., not significant. 


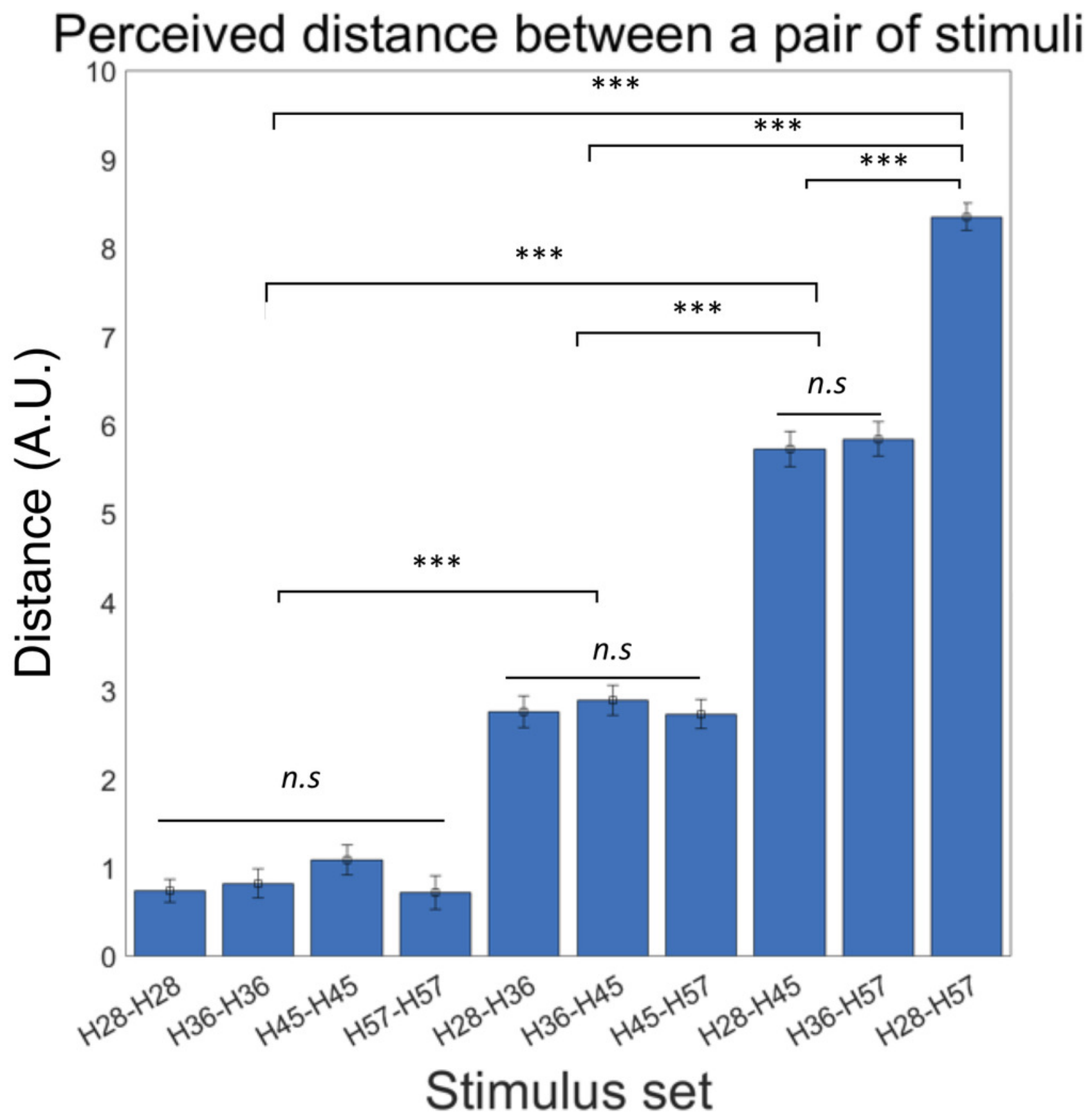




\section{Figure 4}

Neural responses in the posterior insula in the right hemisphere (rplns) positively track physical hardness intensity.

(A) A network of brain regions including rplns positively tracked perceptual hardness intensity of stimuli ( $T$ activation map from parametric modulation, family-wise error $p<0.05$; displayed at $p<0.001$, cluster size $>10$ for illustrative purposes). (B) For illustrative purposes, we estimated percent signal change (PSC) in the rplns region of interest (ROI). PSC shows that the blood oxygen-level dependent responses in the ROI positively tracked the physical hardness intensity of stimuli. There were a set of brain regions that negatively tracked physical hardness intensity (Fig 4A). We found a significant negative correlation in the right posterior lobe ofthe cerebellum (rpCerebellum) (MNI space coordinates of $x=42, y$ $=-74, z=-40 ;$ FWE corrected $p<0.05$, cluster size $>10$, cluster defining threshold $p<$ 0.001) (Fig 4A).
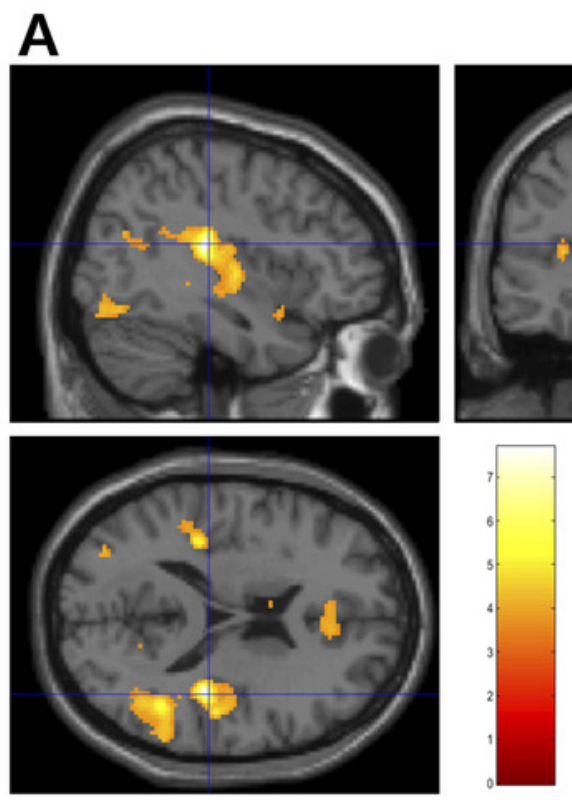

B

T-value

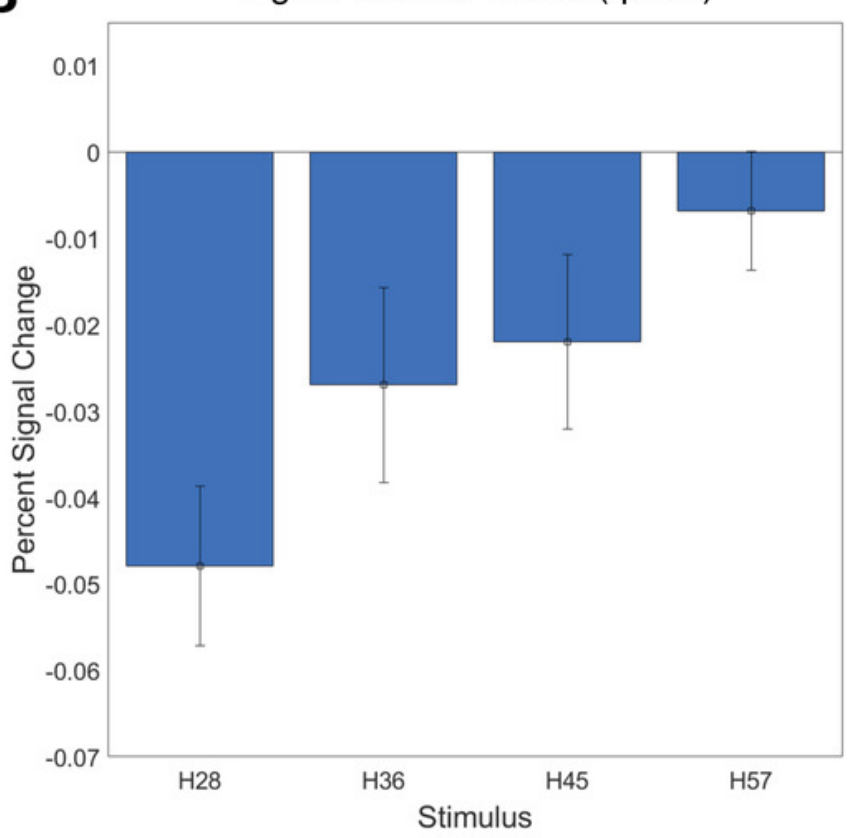




\section{Figure 5}

Neural responses in the right posterior lobe of the cerebellum (rpCerebellum) negatively track physical hardness intensity.

(A) rpCerebellum negatively tracked perceptual hardness intensity of stimuli (T activation map from parametric modulation, family-wise error $p<0.05$; displayed at $p<0.001$, cluster size $>10$ for illustrative purposes). (B) For illustrative purposes, we estimated percent signal change (PSC) in the cerebellum region of interest (ROI). PSC shows that the blood oxygenlevel dependent responses in the ROI negatively tracked the physical hardness intensity of stimuli.
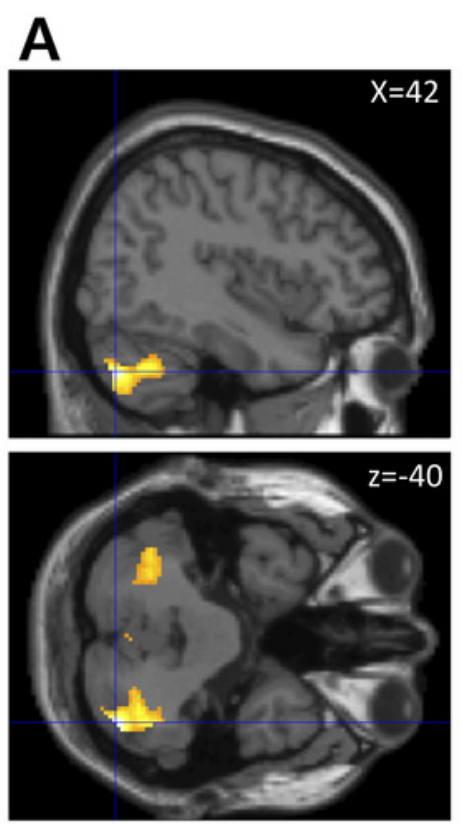
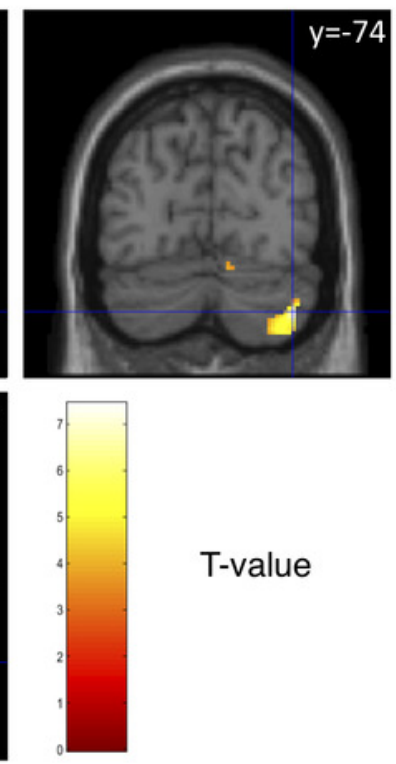

B

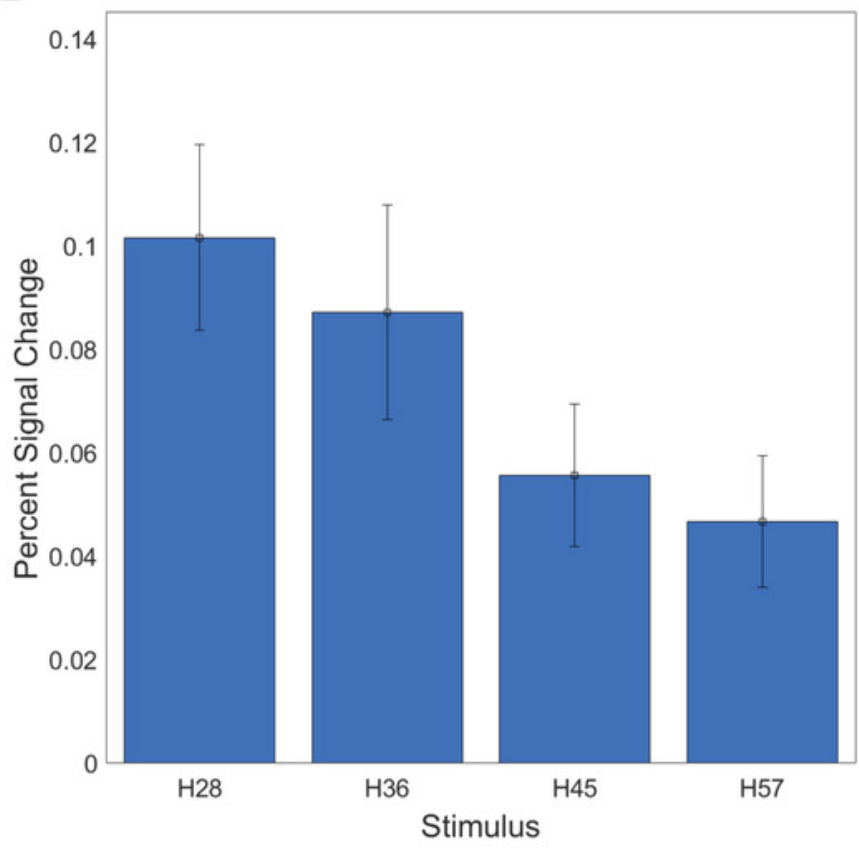




\section{Figure 6}

Dissimilarity matrix of the stimuli based on physical, perceptual hardness, and neural data.

Measurements of physical, perceptual, and neural differences between all possible pairs of four stimuli ( $\mathrm{H} 28, \mathrm{H} 36, \mathrm{H} 45$ and $\mathrm{H} 57)$ produced three symmetric dissimilarity matrices (see text for difference measures). The color bar illustrates differences in the normalized scale from 0 to 10 .

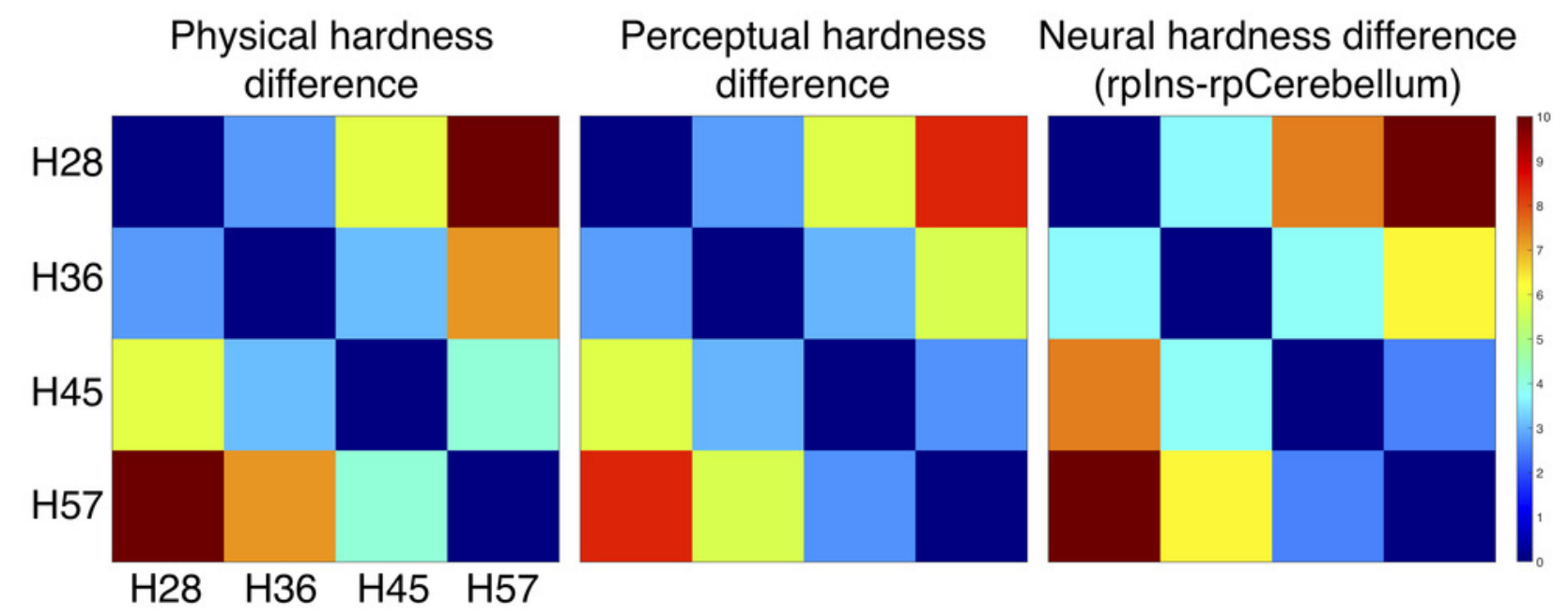


Figure 7

Multidimensional scaling results.

The relative positions of the four different types of hardness intensities across physical, perceptual, and neural properties are depicted in two-dimensional space.

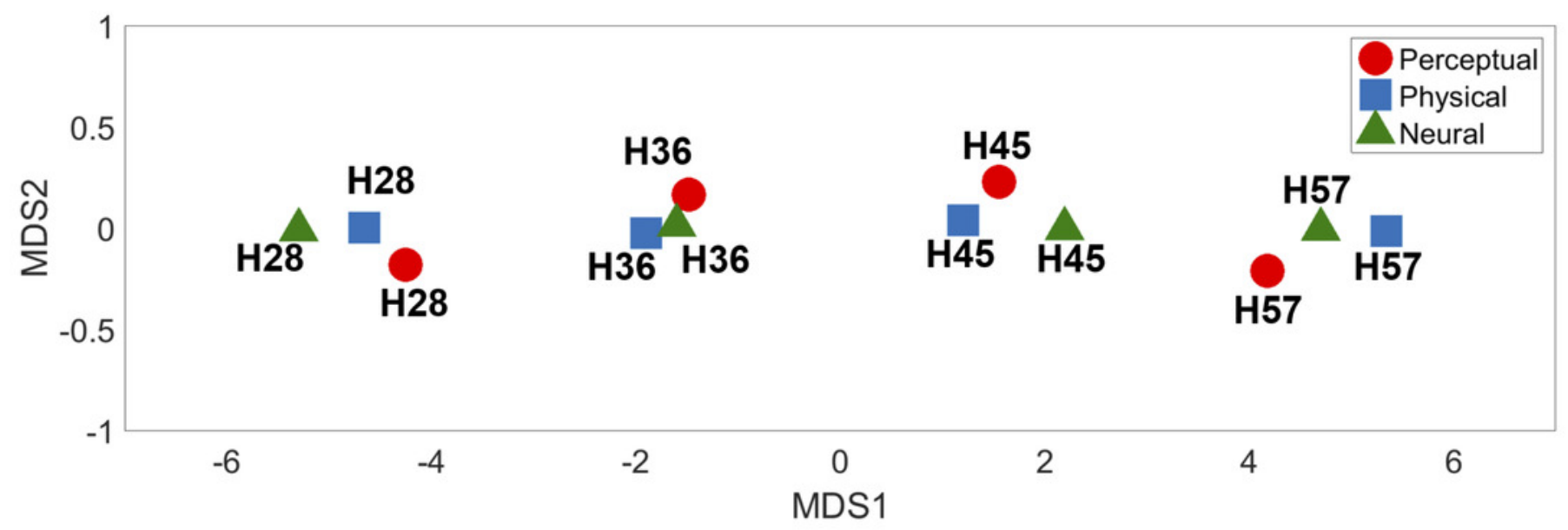

